\title{
Organic chemistry in circumstellar envelopes: Setting the stage for prebiotic synthesis
}

\author{
Lucy M. Ziurys \\ Depts. of Chemistry and Astronomy, Arizona Radio Observatory, Laplace Center for \\ Astrobiology, and Steward Observatory, University of Arizona \\ 933 N. Cherry Ave. Tucson, AZ 85721 USA \\ email: lziurys@as.arizona.edu
}

\begin{abstract}
One of the few carbon-rich environments found in interstellar space is the ejecta of asymptotic giant branch (AGB) stars. Such material, which forms a circumstellar envelope, becomes enriched in carbon due to "dredge-up" phenomena associated with nucleosynthesis. A unique organic synthesis flourishes in the gas phase in these envelopes, and radio and millimeter observations have identified a wide range of C-bearing compounds, including long acetylenic chains such as $\mathrm{HC}_{5} \mathrm{~N}, \mathrm{HC}_{7} \mathrm{~N}, \mathrm{C}_{4} \mathrm{H}, \mathrm{C}_{6} \mathrm{H}, \mathrm{C}_{8} \mathrm{H}, \mathrm{C}_{6} \mathrm{H}^{-}, \mathrm{C}_{8} \mathrm{H}^{-}$, and $\mathrm{C}_{3} \mathrm{O}$. Oxygen-rich envelopes also have a non-negligible carbon chemistry, fostering species such as $\mathrm{HCN}$ and $\mathrm{HCO}^{+}$. Phosphorus chemistry appears to be active as well in circumstellar shells, as evidenced by the recent detections of HCP, CCP, and PO. Radio observations also indicate that some fraction of the circumstellar molecular material survives into the planetary nebula stage, and then becomes incorporated into diffuse, and eventually, dense clouds. The complex organic molecules found in dense clouds such as Sgr B2(N) may be the products of "seed" material that can be traced back to the carbon-enriched circumstellar gas.
\end{abstract}

Keywords. Circumstellar matter, stars: AGB and post-AGB, astrochemistry, astrobiology, submillimeter, ISM: abundances

\section{Introduction}

Although the concept of Darwinian evolution implies that the "best" solutions were attained in living systems, there are in fact many biochemical paradoxes. For example, why are only twenty amino acids used in proteins in living organisms on Earth? A wide range of experiments have shown that "non-natural" amino acids can be readily incorporated into proteins (e.g., Hohsaka \& Sisido 2002). Why does genetics only use the sugars ribose and deoxyribose in RNA and DNA? Studies by Eschenmoser and others have demonstrated that other sugars have been found to work equally well in DNA analogs (Eschenmoser 1999). Why only four nucleobase pairs in DNA and RNA? There are other base pairs that appear to function as satisfactorily, and have been successfully incorporated into enzymes (Yang et al. 2007). Other examples exist, all pointing to the probable situation that life evolved too rapidly on Earth to sample all chemical possibilities (e.g., Benner et al. 2004). Therefore, modern biochemistry likely reflects what starting materials happened to be present on Earth when life began - a matter of "synthetic contingency".

Isotopic studies of noble gases in the Earth's mantle relative to the current atmosphere suggest that the planet lost most of its original carbon in the form of $\mathrm{CH}_{4}, \mathrm{CO}$, and $\mathrm{CO}_{2}$. This loss occurred when Earth shed its original atmosphere (Harper \& Jacobsen 1996). The organic material that was incorporated into living systems probably originated from 
somewhere other than early Earth. It is clear that our planet has been bombarded by meteorites, comets, and interplanetary dust particles since its formation, with particularly heavy bombardment 3.8-4.0 billion years ago (Gomes et al. 2005). Shortly thereafter, life is thought to have developed (Furnes et al. 2004). It has been suggested for some time that exogenous delivery of organics to Earth during this period may have provided the necessary carbonaceous material that gave a "jump-start" to life (Anders 1989, Chyba \& Sagan 1992). The organic matter brought by meteorites, comets, and dust particles probably had in part an interstellar origin, as suggested by isotopic studies (e.g., Pizzarello et al. 2008, Keller et al. 1994, Mumma et al. 2002). Where did this material originate?

\section{The Role of Circumstellar Envelopes Around Evolved Stars}

The origin of carbon lies in nucleosynthesis in stars. There are two main contributors to carbon, supernovae $\left({ }^{12} \mathrm{C}\right)$ and evolved stars $\left({ }^{12} \mathrm{C}\right.$ and $\left.{ }^{13} \mathrm{C}\right)$, including red giants, supergiants, and asymptotic giant branch (AGB) stars. Stellar yields from such objects result in an average galactic $\mathrm{C} / \mathrm{O}$ ratio of 0.7 (Carigi et al. 2005). Hence, in most stars, $\mathrm{C}<\mathrm{O}$. The exceptions are AGB stars, which commonly have $\mathrm{C}>\mathrm{O}$.

The AGB phase is an evolutionary stage for intermediate mass stars. Such stars begin their life cycle on the main sequence with $\mathrm{C}<\mathrm{O}$. When hydrogen in the stellar core becomes depleted, it contracts, causing a rise in temperature that ignites a surrounding shell. The outer stellar envelope then expands and cools, creating a red giant star. The envelope of a red giant becomes very convective, and mixes by-products of hydrogenburning nucleosynthesis to the surface, including ${ }^{13} \mathrm{C}$, in a phenomenon termed "first dredge-up" (Herwig 2006). The first dredge-up increases the abundance of ${ }^{13} \mathrm{C}$ relative to ${ }^{12} \mathrm{C}$. Stellar collapse continues and finally helium in the core ignites. When this supply is exhausted, nucleosynthesis continues in He- and H-burning shells around a $\mathrm{C} / \mathrm{O}$ core, and the AGB begins. Now the convective atmosphere dips into the helium-burning shell and brings up nucleosynthetic products to the stellar surface, the "third dredge-up". The main product of He-burning is ${ }^{12} \mathrm{C}$. Therefore, this dredge-up event converts an initially oxygen-rich star to a carbon-rich one, in the process raising the effective ${ }^{12} \mathrm{C} /{ }^{13} \mathrm{C}$ ratio.

The change from an environment with $\mathrm{C}<\mathrm{O}$ to $\mathrm{C}>\mathrm{O}$ as a result of mixing of the He-burning product ${ }^{12} \mathrm{C}$ is routinely observed in evolved stars. H-burning results in large amounts of ${ }^{13} \mathrm{C}$, while He-burning enhances ${ }^{12} \mathrm{C}$. As shown in Figure 1, the ${ }^{12} \mathrm{C} /{ }^{13} \mathrm{C}$ ratio measured in stellar ejecta, is low $\left({ }^{12} \mathrm{C} /{ }^{13} \mathrm{C}<50\right)$ for O-rich stars. As the objects evolve onto the $\mathrm{AGB}$, their $\mathrm{C} / \mathrm{O}$ ratio increases, as does their ${ }^{12} \mathrm{C} /{ }^{13} \mathrm{C}$ ratio.

During the red giant phase, stars begin to loose their outer atmosphere, which creates a cool circumstellar envelope (e.g. Kwok 2004). This mass loss increases in the AGB phase, where as much as $10^{-4}$ solar masses per year are being ejected. Consequently, a massive envelope is created around these objects in which $\mathrm{C}>\mathrm{O}$. In this unique environment, an unusual organic chemistry occurs.

\section{Organic Chemistry in Circumstellar Gas}

The chemistry of circumstellar shells has been a subject of astronomical observations for many decades, particularly at radio and millimeter wavelengths. Such observations have focused principally on C-rich AGB stars, in particular IRC+10216, as well as the proto-planetary nebulae CRL 618 and CRL 2688. A great deal has been learned from broad-band spectral line surveys of these objects, such as the IRAM $2 \mathrm{~mm}$ survey (Cernicharo et al. 2000) and the Nobeyama $\mathrm{K}_{a}$ band survey (Kawaguchi et al. 1995), both of IRC+10216. More recent observations include millimeter band scans of CRL 618 (Pardo et al. 2007), and of the oxygen-rich supergiant VY Canis Majoris, or VY 


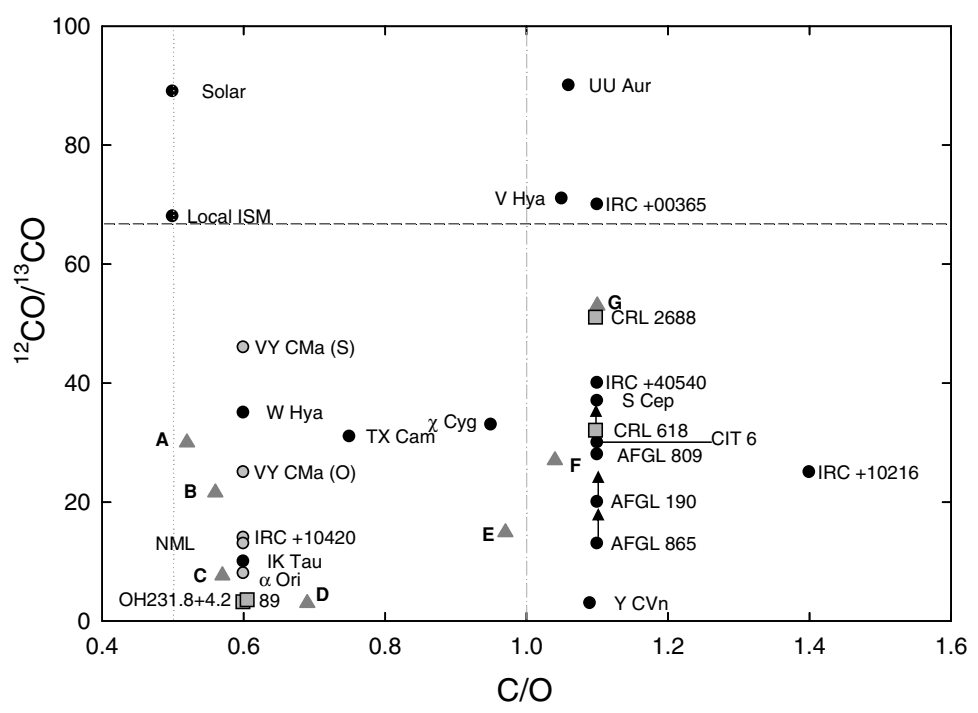

Figure 1. Circumstellar ${ }^{12} \mathrm{C} /{ }^{13} \mathrm{C}$ ratios from $\mathrm{CO}$ vs. $\mathrm{C} / \mathrm{O}$ ratios for a sample of nearby evolved stars (Milam et al. 2008a). A through G are model calculations. The large arrow shows the trend.

CMa (Tenenbaum et al. 2008a), conducted with the IRAM $30 \mathrm{~m}$ and the Arizona Radio Observatory (ARO) Sub-Millimeter Telescope (SMT), respectively. For the carbon-rich circumstellar shells, the results have been striking. On the order of 56 carbon-bearing molecules have been identified in $\mathrm{C}$-rich envelopes, including a variety of hydrocarbon

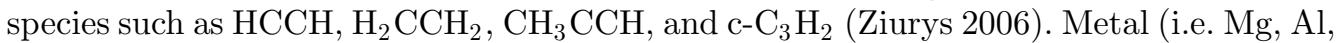
$\mathrm{Na}$ ) and silicon-bearing carbon species are also present. In contrast, only 11 non-carbon containing molecules have been identified in these objects.

Perhaps the most remarkable aspect of this chemistry is the presence of long carbonchains, including $\mathrm{HC}_{n} \mathrm{~N}(n=3-9), \mathrm{C}_{n} \mathrm{H}(n=2-8), \mathrm{C}_{2 n} \mathrm{H}^{-}(n=2-4)$, and $\mathrm{SiC}_{n}(n=1-4)$. Even $\mathrm{C}_{3} \mathrm{O}$ has recently been identified in IRC+10216 (Tenenbaum et al. 2006). The total abundance of carbon chain species, relative to $\mathrm{H}_{2}$, is $f \approx 1.3 \times 10^{-5}$ (Table 1 ); considering that $\mathrm{C} / \mathrm{H}$ is $10^{-4}$, cosmically, a large amount of material is in the form of $\mathrm{C}-\mathrm{C}$ bonds.

Many evolved stars with high mass loss rates remain oxygen-rich. In such environments, it has been thought that all the carbon should be in the form of CO (e.g. Mamon et al. 1987). Recent observations by Ziurys et al. (2007, 2008) have shown that even in such O-rich shells, carbon chemistry is active. For example, in the O-rich supergiant star VY CMa, six carbon-bearing molecules have been observed, including HCN, CS, HNC, and $\mathrm{HCO}^{+}$(Figure 2).

$\mathrm{HCN}$ and $\mathrm{HCO}^{+}$have also been observed in other O-rich envelopes, such as TX Cam, W Hya, IK Tau, and NML Cyg (Ziurys et al. 2008, Pulliam \& Ziurys 2008). Typical abundances for $\mathrm{HCN}$ is $f \approx 10^{-5}$, relative to $\mathrm{H}_{2}$, and $f \approx 10^{-8}$ for $\mathrm{HCO}^{+}$. These molecules are thought to be produced by either photospheric shocks in the inner envelope (Cherchneff 2006), or by photochemistry in the outer shell (Mamon et al. 1987).

\section{Circumstellar Phosphorus Chemistry}

As an element, phosphorus is the 18th most abundant, cosmically, yet it is the fifth most important biologically (Pasek \& Lauretta 2005). Because of its relatively low abundance, it has been thought to play a minor role in interstellar chemistry. Thus far, only one phosphorus-bearing molecule has been observed in molecular clouds, PN (Ziurys 
Table 1. Abundances of Carbon-Chain Molecules in IRC+10216

\begin{tabular}{cccc}
\hline Molecule & $T_{\text {rot }}(\mathrm{K})$ & $N_{\text {tot }}\left(\mathrm{cm}^{-2}\right)$ & $f\left(\mathrm{X} / \mathrm{H}_{2}\right)$ \\
$\mathrm{CCH}$ & 20 & $5 \times 10^{15}$ & $7 \times 10^{-6}$ \\
$\mathrm{l}^{-} \mathrm{C}_{3} \mathrm{H}$ & 20 & $7 \times 10^{13}$ & $1 \times 10^{-8}$ \\
$\mathrm{C}_{4} \mathrm{H}$ & 35 & $3 \times 10^{15}$ & $4 \times 10^{-6}$ \\
$\mathrm{C}_{5} \mathrm{H}$ & 25 & $4 \times 10^{13}$ & $6 \times 10^{-8}$ \\
$\mathrm{C}_{6} \mathrm{H}$ & 35 & $6 \times 10^{13}$ & $8 \times 10^{-8}$ \\
$\mathrm{C}_{7} \mathrm{H}$ & 35 & $2 \times 10^{12}$ & $3 \times 10^{-9}$ \\
$\mathrm{C}_{8} \mathrm{H}$ & 52 & $1 \times 10^{13}$ & $1 \times 10^{-8}$ \\
$\mathrm{C}_{3} \mathrm{~N}$ & 20 & $3 \times 10^{14}$ & $4 \times 10^{-7}$ \\
$\mathrm{C}_{5} \mathrm{~N}$ & 35 & $6 \times 10^{12}$ & $9 \times 10^{-9}$ \\
$\mathrm{C}_{3} \mathrm{O}$ & 27 & $1.2 \times 10^{12}$ & $2 \times 10^{-9}$ \\
$\mathrm{C}_{4} \mathrm{H}^{-}$ & 23 & $7.1 \times 10^{11}$ & $9.5 \times 10^{-8}$ \\
$\mathrm{C}_{6} \mathrm{H}^{-}$ & 27 & $4.1 \times 10^{12}$ & $5.5 \times 10^{-9}$ \\
$\mathrm{C}_{8} \mathrm{H}^{-}$ & 16 & $2.6 \times 10^{12}$ & $3.7 \times 10^{-9}$ \\
$\mathrm{HC}_{3} \mathrm{~N}$ & 35 & $2 \times 10^{15}$ & $7 \times 10^{-7}$ \\
$\mathrm{HC}_{5} \mathrm{~N}$ & 12 & $4 \times 10^{14}$ & $1.5 \times 10^{-7}$ \\
$\mathrm{HC}_{7} \mathrm{~N}$ & 12 & $1 \times 10^{14}$ & $6 \times 10^{-8}$ \\
$\mathrm{HC}_{9} \mathrm{~N}$ & 12 & $2.3 \times 10^{13}$ & $7.7 \times 10^{-9}$ \\
\hline
\end{tabular}

From Cernicharo et al. 2000, 2007, Truong-Bach et al. 1993, Tenenbaum et al. 2006, Remijan et al. 2007

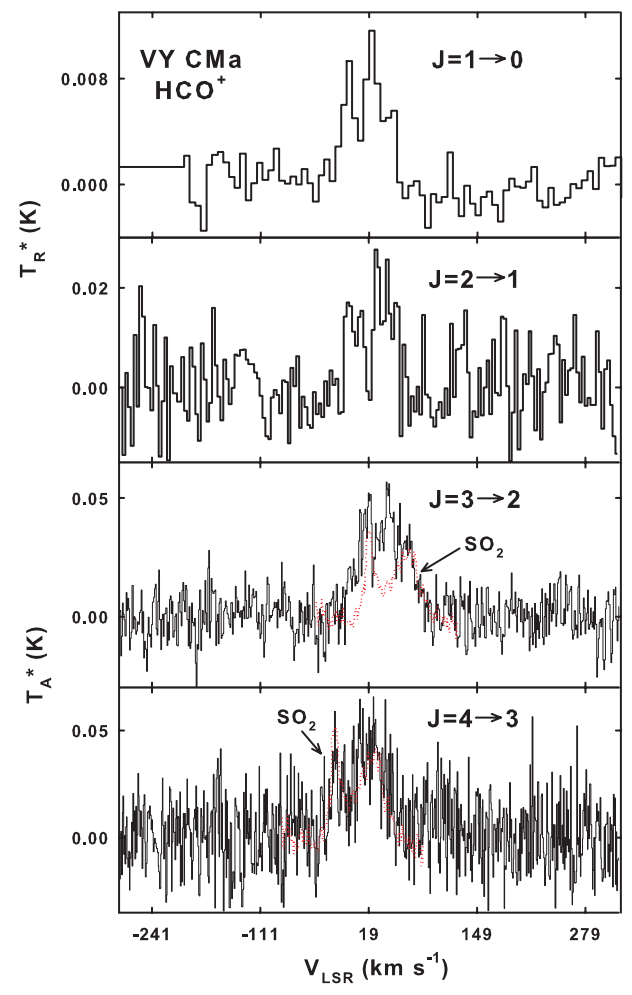

Figure 2. Spectra of $\mathrm{HCO}^{+}$observed towards VY CMa using the ARO $12 \mathrm{~m}$ and SMT (Ziurys et al. 2008). The $J=3 \rightarrow 2$ and $4 \rightarrow 3$ transitions are blended with lines of $\mathrm{SO}_{2}$. 
1987, Turner et al. 1990). For many years, the only phosphorus-containing species identified in circumstellar gas has been CP, observed in IRC+10216 (Guélin et al. 1990). Very recent studies, however, have changed this perception. Within the past year, HCP, $\mathrm{PO}, \mathrm{PN}$, and most recently, $\mathrm{CCP}$ have been detected in circumstellar gas. PO, the first molecule observed in space with a phosphorus-oxygen bond, has been found in VY CMa using the ARO SMT (Tenenbaum et al. 2007). PN and HCP have been identified in both IRC+10216 and CRL 2688 (Agúndez et al. 2007, Milam et al. 2008). In the case of CCP, new laboratory spectroscopic measurements were critical for its identification (Halfen et al. 2008). CCP has a ${ }^{2} \Pi$ ground electronic state and therefore each rotational transition is split into lambda-doublets. The doublet structure measured in the laboratory is evident in the astrophysical spectrum, measured towards IRC+10216 using the ARO $12 \mathrm{~m}$ (Figure 3).

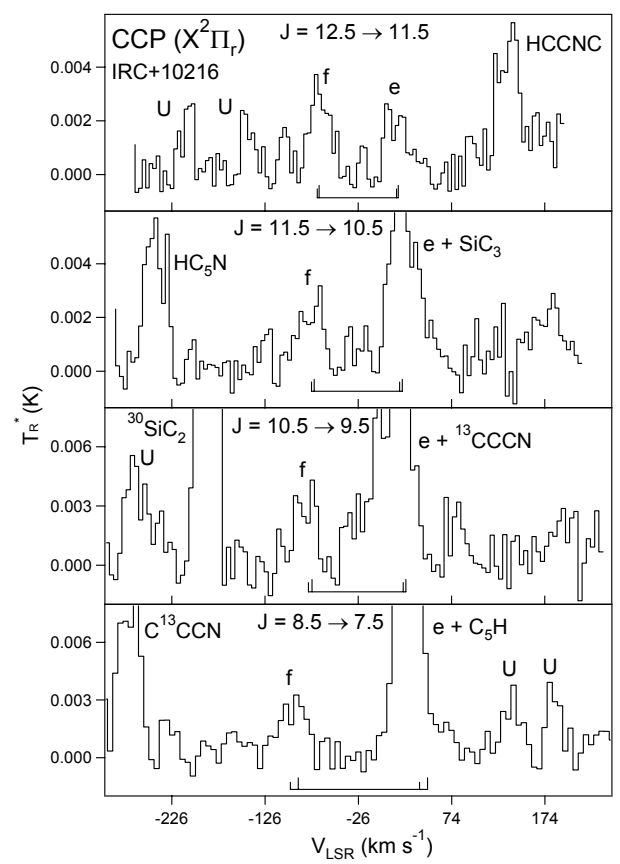

Figure 3. Spectra of CCP observed towards IRC+10216. The lines underneath the data show the positions and relative intensities of the lambda-doublets that make up each rotational transition (Halfen et al. 2008).

A current summary of the abundances of circumstellar phosphorus-containing compounds is given in Table 2. This table reflects the limited observations conducted thus far. HCP appears to be the most abundant of these compounds in C-rich shells, and may be formed by LTE chemistry close to the stellar photosphere (Agúndez et al. 2007). $\mathrm{PO}$ is important for O-rich objects, while PN is common to both types of envelopes. CP and CCP have only been identified in IRC +10216 , where they arise exclusively in the outer envelope, as might be expected for free radicals. HCP may be the precursor species for these two organophosphorus compounds. Fractional abundances for these molecules fall typically in the range $f=10^{-9}-10^{-8}$, relative to $\mathrm{H}_{2}$, with $\mathrm{PN}$ having the lowest value in IRC +10216 of $3 \times 10^{-10}$. About $10 \%$ of the available phosphorus is in gasphase molecules. In contrast, abundances observed for PN in molecular clouds vary from $f=1 \times 10^{-12}$ to $2 \times 10^{-10}$, relative to $\mathrm{H}_{2}$ (Turner et al. 1990). This compound has been identified in five dense, star-forming regions [Orion-KL, Sgr B2, W51M, M17-SW, and 


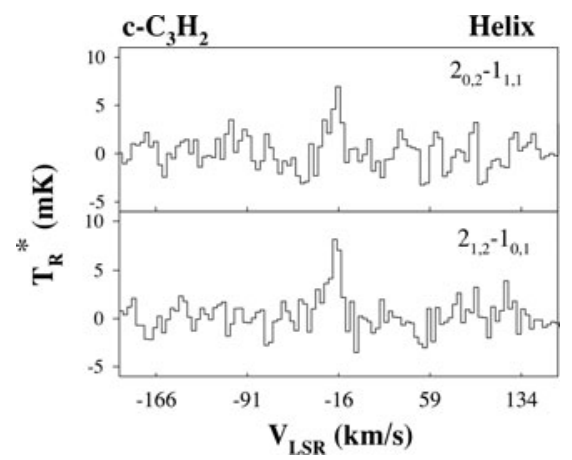

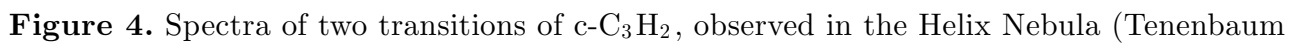
et al. 2008b).

DR-21(OH)], but has yet to be observed in colder, dark clouds. HCP has been sought in these same objects, but not detected with limits of $f<1-4 \times 10^{-10}$.

Table 2. Abundances of Phosphorus-Containing Species in Circumstellar Gas ${ }^{a}$ )

\begin{tabular}{ccccc}
\hline Source & Molecule & $\theta_{s}()$ & $N_{\text {tot }}\left(\mathrm{cm}^{-2}\right)$ & $f\left(\mathrm{X} / \mathrm{H}_{2}\right)$ \\
IRC +10216 & PN & 36 & $1 \times 10^{13}$ & $3 \times 10^{-10}$ \\
& HCP & 22 & $1 \times 10^{15}$ & $3 \times 10^{-8}$ \\
& CP & 26 (shell) & $1 \times 10^{14}$ & $1 \times 10^{-8}$ \\
& CCP & 80 (shell) & $1 \times 10^{12}$ & $1 \times 10^{-9}$ \\
CRL 2688 & PN & $8-20$ & $5-8 \times 10^{15}$ & $3-5 \times 10^{-9}$ \\
& HCP & $8-12$ & $3 \times 10^{17}$ & $2 \times 10^{-7}$ \\
VY CMa & PO & 1 & $2.8 \times 10^{15}$ & $9 \times 10^{-8}$ \\
& PN & 1 & $2.0 \times 10^{15}$ & $4 \times 10^{-8}$ \\
\hline
\end{tabular}

a) From Milam et al. (2008b) and Halfen et al. (2008)

\section{Carbon Chemistry in Planetary Nebulae}

AGB stars evolve into planetary nebulae $(\mathrm{PNe})$, the final stage of their life cycle. By this phase, which lasts about 10,000 years, most of the original stellar mass is lost and forms a residual shell around a hot $(T=100,000 \mathrm{~K})$, exposed star. The star is now a strong emitter of ultraviolet (UV) radiation, and ionizes the surrounding material, as evident in optical images. What becomes of the molecular material from the remnant AGB shell? Under these conditions, destruction via photodissociation is a likely outcome. Yet, astronomical observations by Young et al. (1999) towards the Helix Nebula have demonstrated that $\mathrm{CO}$ is present in a large ring surrounding the central star. Bachiller et al. (1997) also detected $\mathrm{HCO}^{+}, \mathrm{HCN}, \mathrm{HNC}$, and $\mathrm{CN}$ in the Helix. The presence of these compounds in the gas phase is surprising because the Helix Nebula is thought to be at the end of its life cycle. The molecules present in this object have been subjected to intense UV radiation for over 10,000 years; yet the molecules survive.

Tenenbaum et al. (2008b) have recently searched for molecules containing C-C and $\mathrm{C}=\mathrm{O}$ bonds in old planetary nebulae, using the $\mathrm{ARO}$ telescopes in the 1-3 $\mathrm{mm}$ wavelength regime. $\mathrm{CCH}$ was detected in four such nebulae: the Helix, NGC 6781, M4-9, and NGC 6720 (Ring Nebula), via its fundamental transition near $87 \mathrm{GHz} . \mathrm{C}_{3} \mathrm{H}_{2}$, a cyclic molecule, and $\mathrm{H}_{2} \mathrm{CO}$, were also observed in the Helix (Figure 4). The identification of 4 and 5 atom 
molecules suggests that more organic species may be discovered in this source and other planetary nebulae.

Theoretical modeling by Howe et al. (1994) offers an explanation for molecule survival. Their calculations show that instabilities in the stellar wind can create finger-like clumps with densities as high as $10^{5} \mathrm{~cm}^{-3}$. Spitzer images of infrared emission actually show the presence of finger-like dust structures in the Helix (Hora et al. 2006). Such clumps, composed of gas-phase molecules mixed with dust, become self-shielding. Molecules thus survive despite the high radiation field.

\section{Into Diffuse and Dense Clouds, and then to Earth?}

The gas and dust from planetary nebulae eventually disperse into the diffuse interstellar medium, enriching this material with heavy elements. In our galaxy, planetary nebulae supply almost an order of magnitude more interstellar mass than supernovae (e.g., Stanghellini et al. 2000). The fate of the molecular material from such nebulae is critical in understanding the evolution of interstellar chemistry.

The remnant material from planetary nebulae becomes incorporated into diffuse clouds, objects with densities of $1-10 \mathrm{~cm}^{-3}$, primarily traced by atomic hydrogen. The general UV radiation field from ambient starlight is thought to be quite high in diffuse clouds, and models suggest that all polyatomic molecules will be photodissociated (van Dishoeck \& Black 1986). Radio astronomical observations by H. Liszt and R. Lucas (e.g., Liszt et al. 2006), however, have demonstrated that diffuse clouds have an unexpectedly rich molecular content. These authors have detected numerous polyatomic species in diffuse gas towards many lines of sight, measuring absorption spectra against background quasars. This technique has enabled the detection of compounds such as $\mathrm{HCO}^{+}, \mathrm{CCH}$, $\mathrm{HCN}, \mathrm{HNC}, \mathrm{C}_{3} \mathrm{H}_{2}$, and $\mathrm{H}_{2} \mathrm{CO}$ in diffuse clouds. The carbon chain species, $\mathrm{C}_{3}$ has also been observed in these objects (Maier et al. 2001). As shown in Table 3, diffuse clouds and planetary nebulae appear to contain similar sets of molecular species. This remarkable coincidence suggests that the molecular content of diffuse clouds may be remnant material from the winds of planetary nebulae. It is difficult to explain diffuse cloud abundances otherwise; the densities in these objects are too low for efficient molecule production, and UV radiation also hinders build-up of polyatomic species. The diffuse cloud abundances are about a factor of 10-100 lower than in the oldest nebulae [M4-9, NGC 7293 (Helix)], consistent with a scheme of partial destruction.

Table 3. Molecules Common to Diffuse Clouds and PNe

\begin{tabular}{cccc}
\hline $\mathrm{CO}$ & $\mathrm{HCO}^{+}$ & $\mathrm{CCH}$ & $\mathrm{OH}$ \\
$\mathrm{CN}$ & $\mathrm{H}_{2} \mathrm{CO}$ & $\mathrm{HCN}$ & $\mathrm{CO}^{+}$ \\
$\mathrm{H}_{2}$ & $\mathrm{c}^{-} \mathrm{C}_{3} \mathrm{H}_{2}$ & $\mathrm{HNC}$ & $\mathrm{CH}^{+}$ \\
$\mathrm{CH}$ & $\mathrm{C}_{3}$ & & \\
& & & \\
\hline
\end{tabular}

Diffuse clouds gravitationally collapse over a period of about $10^{6}$ years into dense clouds, where a robust gas-phase organic chemistry occurs. All basic organic functional groups are present in known gas-phase interstellar molecules in such clouds. Furthermore, the limit of chemical complexity has yet to be established in these objects, and species with four or more carbon atoms are likely to exist. The chemistry in these sources is not combinatorial, however. Selection effects are inherent in interstellar chemical reactions, which take place at low density $\left(n=10^{3}-10^{7} \mathrm{~cm}^{-3}\right)$ and temperature $(T=10-100 \mathrm{~K})$ and cannot attain thermodynamic equilibrium. 
It seems quite difficult to explain the high abundances of organic species with multiple carbon bonds in dense clouds, where, on average, $\mathrm{O}>\mathrm{C}$. However, if molecular material survives from diffuse clouds, and this matter can be traced back to planetary nebulae and subsequently circumstellar envelopes, then the carbon-rich environment of AGB stars may have been partially preserved. Organic chemistry in dense gas may have received a "jump start" from the presence of multiple C-C bonds left over from circumstellar gas. In due course, dense clouds collapse into pre-solar nebulae, and organic material coalesces into small Solar System bodies such as meteorites, IDPs, and comets. Exogenous delivery brings this organic matter to planetary surfaces. Ultimately, it is the preservation of carbon-enriched molecular material from AGB stars that enables organic chemistry to occur. Interstellar chemistry may hold the answers to the biochemical paradoxes of living systems.

This research is supported by NSF grant AST-0607803 and by the NASA through the Astrobiology Institute under Cooperative Agreement No. CAN-02 OSS02.

\section{References}

Agúndez, M., Cernicharo, J., \& Guélin, M. 2007, ApJ (Letters), 662, L91

Anders, E. 1989, Nature, 342, 255

Bachiller, R., Forveille, T., Huggins, P. J., \& Cox, P. 1997, A\&A, 324, 1123

Benner, Ricardo, \& Carrigan 2004, Curr. Opin. Chem. Bio., 8, 672

Carigi, L., Peimbert, M., Esteban, C., \& Garcia-Rojas, J. 2005, ApJ, 623, 213

Cernicharo, J., Guélin, M., Agúndez, M., Kawaguchi, K., McCarthy, M., \& Thaddeus, P. 2007, $A \mathscr{E} A$ (Letters), 467, L37

Cernicharo, J., Guélin, M., \& Kahane, C. 2000, A\&AS, 142, 181

Cherchneff, I. 2006, $A \mathscr{E} A$ A, 456, 1001

Chyba, C. F. \& Sagan, C. 1992, Nature, 355, 125

Eschenmoser, A. 1999, Science, 284, 2118

Furnes, H., Banerjee, N. R., Muehlenbachs, K., Staudigel, H., \& de Wit, M. 2004, Science, 304, 578

Gomes, R., Levison, H. F., Tsiganis, K., \& Morbidelli, A. 2005, Nature, 435, 466

Guélin, M., Cernicharo, J., Paubert, G., \& Turner, B. E. 1990, A\&A (Letters), 230, L9

Halfen, D. T., Clouthier, D. J., \& Ziurys, L. M. 2008, ApJ (Letters), 677, L101

Harper, C. L., Jr., \& Jacobsen, S. B. 1996, Science, 273, 1814

Herwig, F. 2006, Proc. of the Int. Sym.on Nuclear Astrophys. IX. CERN, p. 206

Hohsaka, T. \& Sisido, M. 2002, Curr. Opin. Chem. Bio., 6, 809

Hora, J. L., Latter, W. B., Smith, H. A., \& Marengo, M. 2006, ApJ, 652, 426

Howe, D. A., Hartquist, T. W., \& Williams, D. A. 1994, MNRAS, 271, 811

Kawaguchi, K., Kasai, Y., Ishikawa, S.-I., \& Kaifu, N. 1995, PASJ, 47, 853

Keller, L. P., Thomas, K. L., Clayton, R. N., Mayeda, T. K., Dehart, J. M., \& McKay, D. S. 1994, GeCoA, 58, 5589

Kwok, S. 2004, Nature, 430, 985

Liszt, H., Lucas, R., \& Pety, J. 2006, A\&SA, 448, 253

Maier, J. P., Lakin, N. M., Walker, G. A. H., \& Bohlender, D. A. 2001, ApJ, 553, 267

Mamon, G. A., Glassgold, A. E., \& Omont, A. 1987, ApJ, 323, 306

Milam, S. N., Halfen, D. T., Tenenbaum, E. D., Apponi, A. J., Woolf, N. J., \& Ziurys, L. M. 2008b, ApJ, in press

Milam, S. N., Woolf, N. J., \& Ziurys, L. M. 2008a, ApJ, in press

Mumma, M. J., Disanti, M. A., dello Russo, N., Magee-Sauer, K., Gibb, E., \& Novak, R. 2002, Proceedings of Asteroids, Comets, Meteors, p. 753

Pardo, J. R., Cernicharo, J., Goicoechea, J. R., Guélin, M., \& Ramos, A. A. 2007, ApJ, 661, 250

Pasek, M. A. \& Lauretta, D. S. 2005, Astrobiology, 5, 515

Pizzarello, S., Huang, Y., \& Alexandre, M. R. 2008, in press

Pulliam, R. \& Ziurys, L. M. 2008, in prep. 
Remijan, A. J., Hollis, J. M., Lovas, F. J., Cordiner, M. A., Millar, T. J., Markwick-Kemper, A. J., \& Jewell, P. R. 2007, ApJ (Letters), 664, L47

Stanghellini, L., Shaw, R. A., Balick, B., \& Blades, J. C. 2000, ApJ (Letters), 534, L167

Tenenbaum, E. D., Apponi, A. J., Ziurys, L. M., Agúndez, M., Cernicharo, J., Pardo, J. R., \& Guélin, M. 2006, ApJ (Letters), 649, L17

Tenenbaum, E. D., Milam, S. N., Apponi, A. J., Woolf, N. J., Ziurys, L. M., \& Shóier, F. 2008a, in prep.

Tenenbaum, E. D., Milam, S. N., Xilouris, K., Woolf, N. J., \& Ziurys, L. M. 2008b, in prep.

Tenenbaum, E. D., Woolf, N. J., \& Ziurys, L. M. 2007, ApJ (Letters), 666, L29

Truong-Bach, Graham, D., \& Nguyen-Q-Rieu 1993, A\& A, 277, 133

Turner, B. E., Tsuji, T., Bally, J., Guélin, M., \& Cernicharo, J. 1990, ApJ, 365, 569

van Dishoeck, E. F. \& Black, J. H. 1986, ApJS, 62, 109

Yang, Z., Sismour, A. M., Sheng, P., Puskar, N. L., \& Benner, S. A. 2007, Nucleic Acids Res., 35,4238

Young, K., Cox, P., Huggins, P. J., Forveille, T., \& Bachiller, R. 1999, ApJ, 522, 387

Ziurys, L. M. 1987, ApJ (Letters), 321, L81

Ziurys, L. M. 2006, PNAS, 103, 12274

Ziurys, L. M., Milam, S. N., Apponi, A. J., \& Woolf, N. J. 2007, Nature, 447, 1094

Ziurys, L. M., Tenenbaum, E. D., Milam, S. N., \& Woolf, N. J. 2008, ApJ, submitted

\section{Discussion}

ZINNER: What can you say about the carbon chemistry in the atmospheres of WolfRayet stars? Due to the high C/O ratio and the lack of hydrogen, WC stars can have compounds like CP, CCP and WN stars can produce NP.

ZIURYS: So far the study of circumstellar chemistry has been concentrated on a few AGB stars but the observations of WR stars should be very interesting.

NitTLER: Is it possible to measure the carbon isotopes of these molecules in the diffuse ISM? In this way, we may be able to test whether the molecules in diffuse ISM come from AGB stars.

ZIURYS: Yes, that's a very good idea. I think Harvey [Liszt] has measured ${ }^{13} \mathrm{CO}$, but I don't recall him publishing a ${ }^{12} \mathrm{C}:{ }^{13} \mathrm{C}$ ratio. This would be a very interesting thing to do.

VAN DishoECK: I want to comment on the suggestion of bringing this carbon bearing molecules into the diffuse ISM. From the photodissociation rates, the lifetimes of many small carbonaceous molecules including $\mathrm{C}_{3}, \mathrm{C}_{2} \mathrm{H}$ and $\mathrm{C}_{3} \mathrm{H}_{2}$ etc., under diffuse interstellar radiation are all very rapid, of the order of 10 years.

ZIURYS: If these molecules survive in $10^{5}$ particles per cc clumps and they are shielded by dust, then they are not really in a diffuse gas any more. May be there is a symbiotic relationship with the dust, in the sense that while the molecules are being destroyed, they are also being produced. Since Harvey [Liszt] does not see line emission, and only sees absorption against background quasars, he can determine the gas density to be about 10 particles per cc. It's diffuse gas.

SARRE: My first comment is that one can make optical measurements of the ${ }^{12} \mathrm{C}$ to ${ }^{13} \mathrm{C}$ ratio, for example through observations of $\mathrm{CH}^{+}$in diffuse clouds. My second question is what fraction of the carbon budget these molecules in the Helix Nebula take up given that planetary nebulae also contain an aromatic dust component. 
ZiURYs: If we go from AGB circumstellar envelopes to planetary nebula to the diffuse ISM, molecular abundances slowly go down, maybe in order of magnitude per step.

SARRE: So the carbon content may be increasingly in forms such as large molecules or dust.

ZiURYs: The molecules are not confined to the gas phase. The large molecules may be also be fragmenting and there is undoubtedly gas-grain interactions.

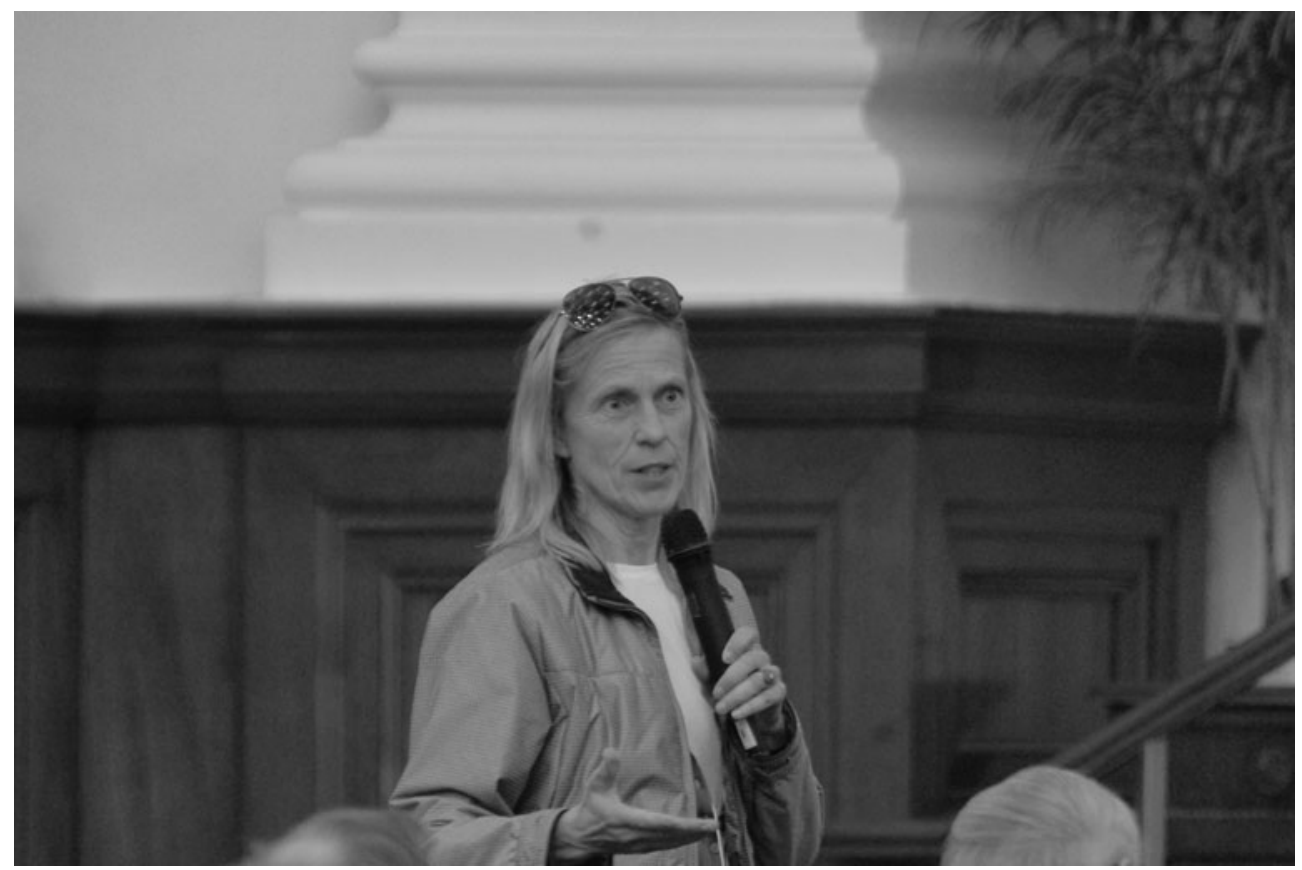

Lucy Ziurys asking one of her many questions (photo by Sze-Leung Cheung). 